\title{
Editorial
}

\section{Preservation of left ventricular function in mitral valve surgery}

Mitral valve surgery aims to correct haemodynamics without causing a deterioration in left ventricular function. An increased awareness that left ventricular function deteriorates after chordal transection or loss of annulo-ventricular continuity after valve replacement has led to an emphasis on repair ${ }^{1-2}$ or replacement methods that preserve annulo-ventricular continuity. ${ }^{3-5}$ This emphasis on preservation of left ventricular structure and function has improved both the early and long-term results of mitral valve operations. ${ }^{6-8}$

A

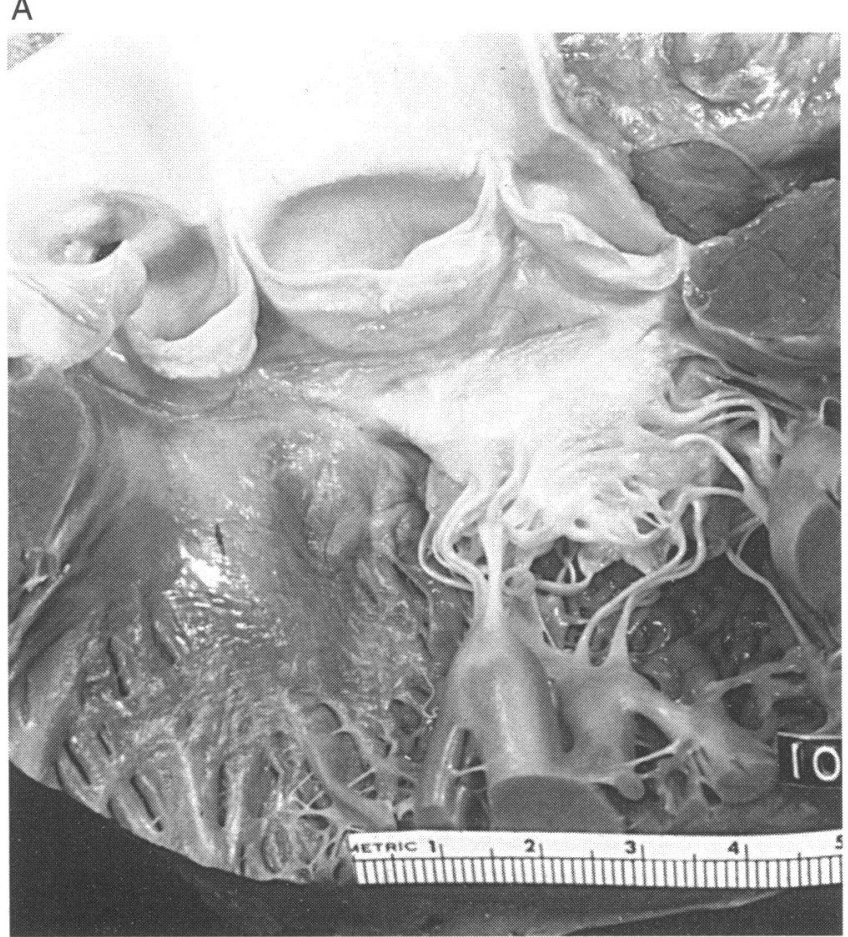

B

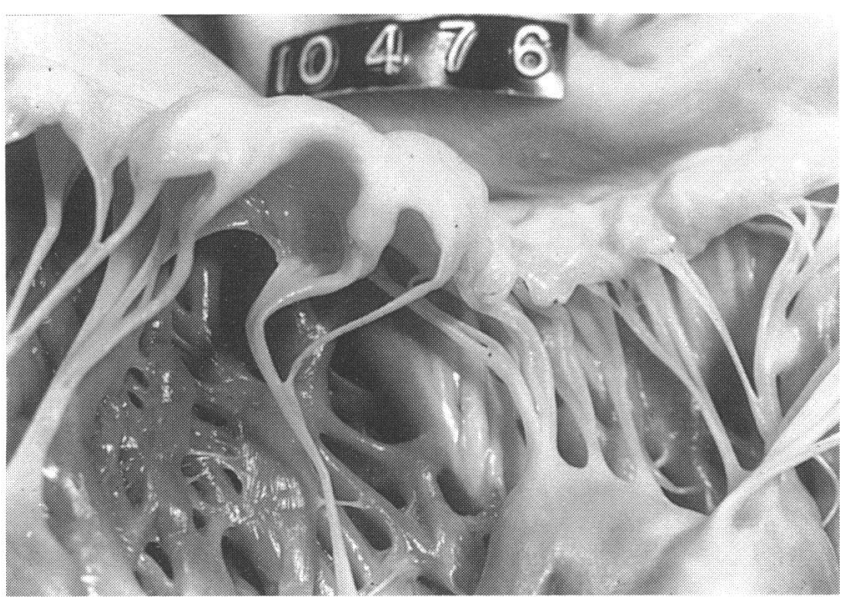

Figure 1 (A) The mitral subvalvar apparatus and relation of the anterior mitral leaflet to the aortic valve. This patient with mitral valve prolapse has elongated chordae tendineae. (B) Detail of chordal attachments to the posterior mitral leaflet. The chordae are elongated with a prolapsing segment.

\section{Aspects of left ventricular structure and function}

The mitral annulus and ventricular wall are both important determinants of left ventricular geometry and function.

In normal hearts the mitral valve orifice is round in diastole and ellipsoidal in systole. During systole the mitral orifice area decreases by $26 \%$ and the circumference decreases by $30 \%$. This change in shape and size is caused by contraction of the bulbospiral muscle bundles (basoconstrictor muscles). ${ }^{9}$

The chordal insertions into both the anterior the posterior leaflets are either into the free edge or into the rough zones on the ventricular surface of the leaflets. The chordae vary in thickness and in the amount of leaflet tissue they support (fig $1 \mathrm{~A}$ and $\mathrm{B}$ ). In the anterior leaflet the chordae insert mainly into the medial and lateral areas, leaving a central clear zone. The papillary muscles are divided into a posteromedial and an anterolateral group each supplied by a central artery derived from the right or left circumflex artery respectively. Each group gives chordae to both leaflets and the number of muscle heads per group is variable.

Canine models have been used to determine the importance of papillary muscle and chordal function in left ventricular mechanics. Gams et al showed that chordal transection at valve replacement in a working dog heart is followed by a significant increase in the longitudinal axis of the left ventricle and a decrease in contractility (fig $2 \mathrm{~A}$ and B). ${ }^{10}$ Left ventricular stroke work is then maintained only at the expense of higher preload and up to $30 \%$ increase in fibre force. Hansen and coworkers found that section of the chordae to the anterior leaflet had a considerably more deleterious effect on left ventricular systolic function than section of the chordae to the posterior leaflet. ${ }^{11}$ Hansen proposed that preservation of the mitral subvalvar apparatus enhances left ventricular systolic performance through reduction of the regional left ventricular afterload. ${ }^{11}$ This optimises the distribution of the myocardial fibre force and allows more synergistic and forceful contraction. The fact that transection of chordae to the anterior leaflet has a more detrimental effect than transection of chordae to the posterior leaflet can be explained by the concept of regional afterload reduction. The anterior leaflet is larger and development of tension in the chordae to this leaflet should be greater at a given left ventricular pressure.

Rushmer initially proposed that interaction between the mitral valve and left ventricular wall through the chordae played an important part in left ventricular dynamics during isovolumetric contraction. ${ }^{12}$ Contraction of the papillary muscles draws the closed mitral valve into the ventricular cavity causing shortening in the longitudinal axis and an increase in the short axis of the left ventricle. This allows the myocardial fibres to stretch more and increases wall tension before contraction (Starling's law). Contraction of the papillary muscles augments the preload of fibres along the circumference of the heart, an effect which is lost by chordal transection. After selective inter- 
A
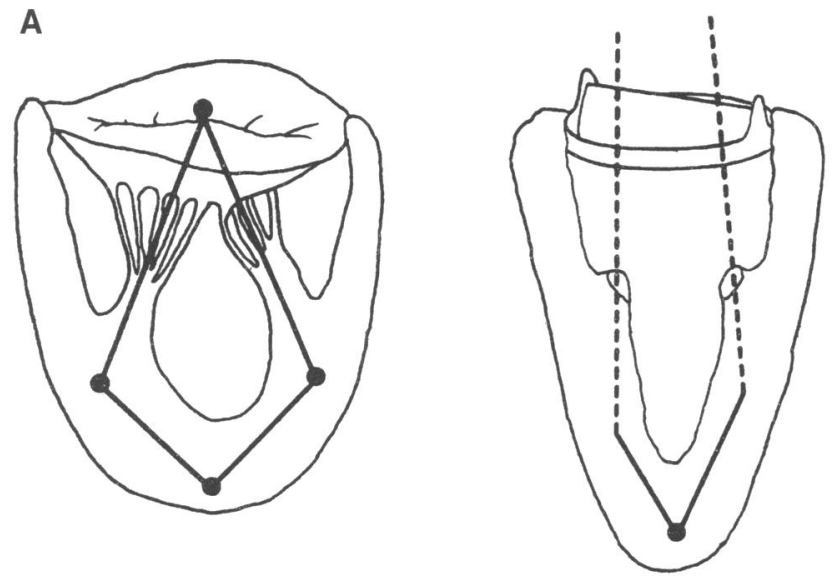

B

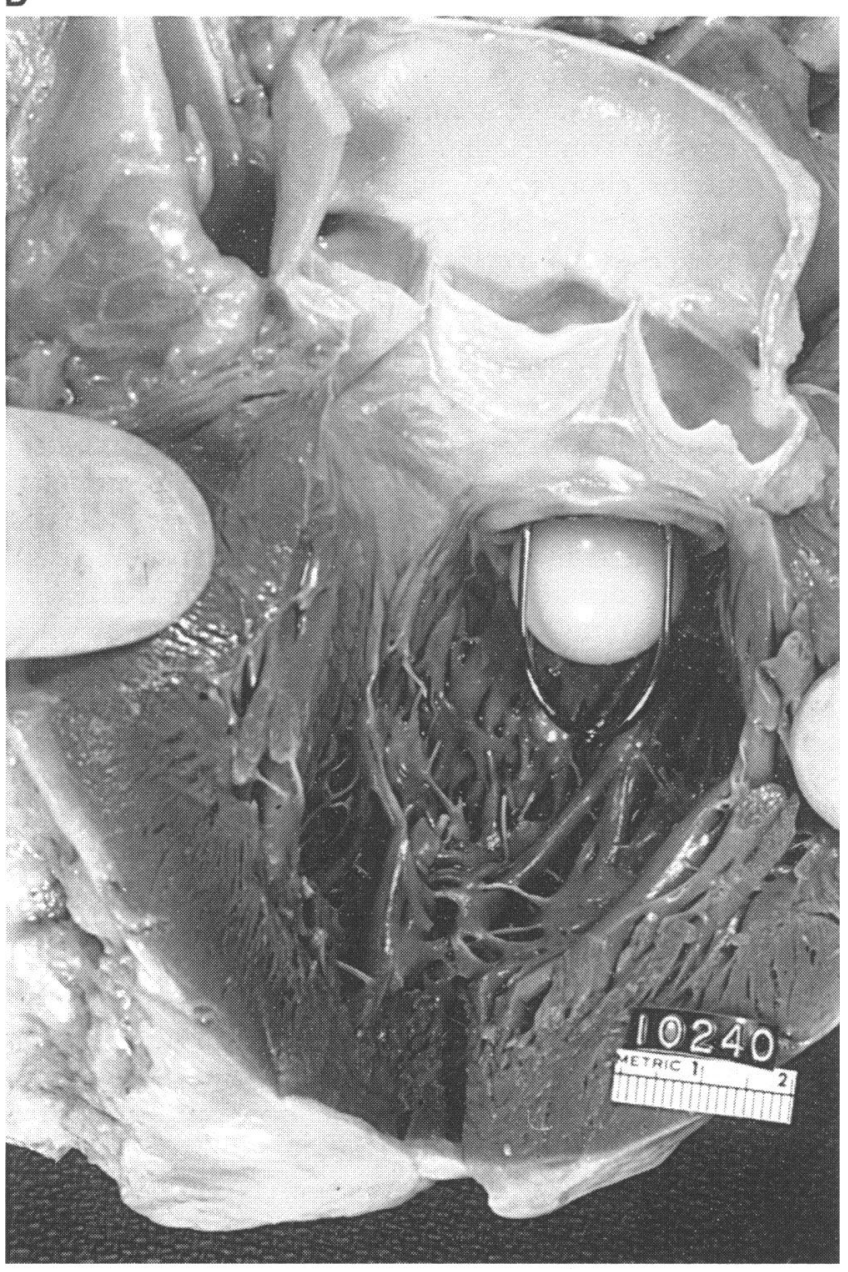

Figure 2 (A) Diagram to show how valve replacement with excision of the subvalvar apparatus results in elongation of the long axis of the left ventricle with a decrease in ejection fraction. Reprinted with the permission of the American Heart Association from Gams E, Heimisch W, Hagl S, et al. Significance of the subvalvular apparatus following mitral valve replacement [abstr]. Circulation 1987;76 suppl IV:538.

(B) Postmortem specimen after mitral valve replacement with a

Starr-Edwards valve. The prosthesis is inherently obstructive and projects into the left ventricular outflow tract. The entire subvalvar apparatus has been excised resulting in dilatation of the left ventricle, impairment of left ventricular function, and failure to gain symptomatic benefit. The patient died from heart failure.

ruption of the chordae, Hansen et al described shortening of the long axis of the ventricle and increase in the minor axis with dilatation of the chamber. ${ }^{11}$ In contrast when the subvalvar mechanism remains intact chamber shape does not change during isovolumetric contraction. In addition chordal section causes areas of dyskinesis at the insertion of the severed papillary muscles, a phenomenon also noted echocardiographically after clinical valve replacement. $^{2}$

\section{Surgery in mitral stenosis}

In rheumatic mitral stenosis where the ventricle remains small and the chordae, papillary muscles, and valve leaflets are fused and rigid with calcification, the annulus loses much or all of its sphincter-like function. Excision of the valve does not have the same effect on ventricular function as in a patient with non-rheumatic degenerative disease and a dilated but contractile mitral annulus. Neither the end systolic nor the end diastolic diameters of the left ventricle appear to change after valve replacement for mitral stenosis. ${ }^{13}$ In contrast, valve excision in patients with degenerative mitral regurgitation has a markedly adverse effect on ventricular diameter and contractility. ${ }^{14} 15$

\section{Surgery in mitral regurgitation}

In chronic mitral regurgitation there is a gradual deterioration of ventricular function. The low impedance leak into the left atrium during systole increases total stroke volume (forward and regurgitant) and ejection fraction. Afterload (systolic wall stress) is increased due to left ventricular dilatation which markedly increases wall tension (Laplace's Law). Surgical correction of mitral regurgitation causes an abrupt increase in left ventricular afterload and adaptation of the left ventricle to this change depends on the integrity of the mitral subvalvar apparatus. ${ }^{16}$

There are now several surgical techniques for the patient with mitral regurgitation. A wide range of repair procedures, including annuloplasty with or without a ring, quadrangular resection of a prolapsed segment, chordal shortening, transposition or replacement, decalcification and pericardial repair are now in use. Valve replacement has traditionally involved chordal transection with excision of the valve. It is now regarded as essential to achieve preservation of the posterior leaflet or bi-leaflet preservation, and a wide choice of mechanical prostheses can be used safely. ${ }^{17-19}$

After mitral valve replacement the left ventricular volume decreases by an amount about equal to the preoperative regurgitant volume. Because left ventricular volume is the major determinant of afterload this falls postoperatively despite an increase in impedance to ejection. After mitral valve replacement with transection of the chordae tendineae the ejection fraction, which is determined by contractility, preload, and afterload, always falls and remains depressed. ${ }^{20}$ The traditional explanation for this fall was that abrupt ablation of the low impedance pathway into the left atrium increased afterload whereas removal of volume overload reduced preload. In reality, the deterioration is probably related to intrinsic abnormalities of myocardial contraction rather than unfavourable loading conditions. In 1964 Lillehei et al introduced the concept of chordal sparing in order to decrease the problems of postoperative low cardiac output syndrome. ${ }^{21}$ The concept was resurrected in 1981 by David who published an experimental study of mitral valve replacement in dogs. ${ }^{22}$ This clearly showed that left ventricular function deteriorated after chordal transections but was unchanged if the chordae tendineae were left intact. David went on to compare resting pre and post operative left ventricular function and exercise-induced changes in patients with isolated chronic mitral regurgitation who had conventional valve replacement (with chordal transection) and in those in whom the continuity between chordae and papillary muscles was preserved. ${ }^{1}$ Left ventricular ejection fraction invariably deteriorated in patients who had excision of 

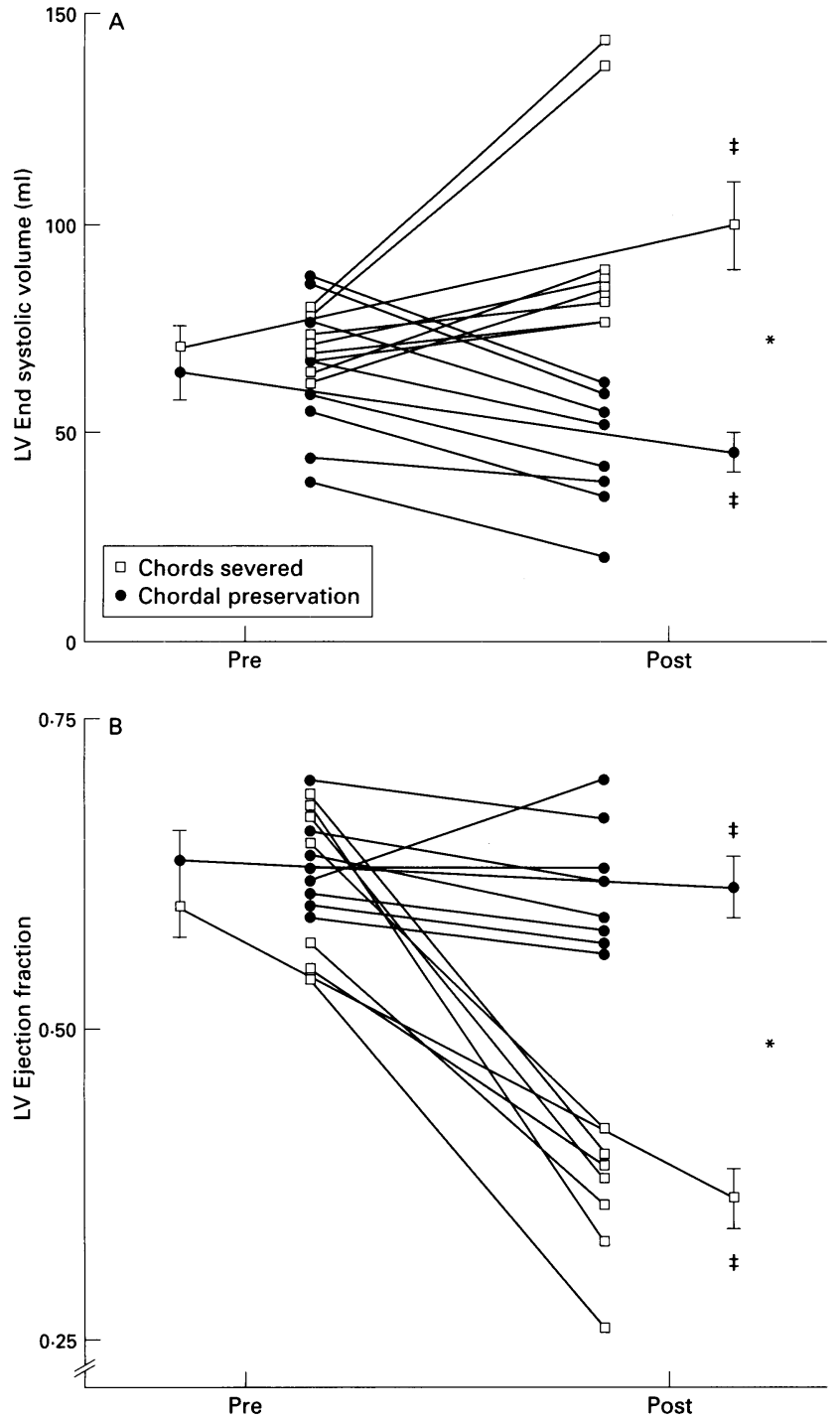

Figure 3 (A) Changes in left ventricular end systolic volume after valve replacement with and without chordal preservation. Chordal transection results in an increase in left ventricular end systolic volume. Reprinted with the permission of the American Heart Association from Rozich TD, Carabello $B A$, Usher $B W$, et al. Mitral valve replacement with and without chordal preservation in patients with chronic mitral regurgitation. Circulation 1992;86:1718-26. (B) Changes in left ventricular ejection fraction in patients with and without chordal preservation. Section of the chordae results in a significant reduction in left ventricular ejection fraction.

the subvalvar apparatus but not in those in whom the chordae were preserved. Left ventricular end diastolic, end systolic, and stroke volume indices decreased significantly in both groups. Cardiac output increased with exercise in both groups through an increase in heart rate, but only those patients with an intact subvalvar apparatus could increase left ventricular ejection fraction and stroke volume index. Although both groups showed a decrease in left ventricular end diastolic and end systolic volume indices the reduction was greater in patients with preserved chordae tendineae. The patients with preserved chordae had better long-term systolic function and left ventricular performance both at rest and during exercise.

In 1979 Miller et al described the surgical technique used to preserve the chordae to the posterior leaflet ${ }^{15}$ and in 1986 David extended this approach by advocating preservation of the anterior leaflet by removal of the quadrilateral central portion (without chordae). ${ }^{23}$ In practice for non-rheumatic valves with a substantial increase in annulus diameter it is possible to roll the entire anterior leaflet into a new annulus without removing any valve tissue. Rozich and colleagues recorded echocardiographic findings and blood pressure measurements in patients before and after correction of chronic mitral regurgitation. ${ }^{24}$ Valve replacement with chordal transection results did not change left ventricle end diastolic volume but considerably increased left ventricular end systolic volume and end systolic stress (fig $3 \mathrm{~A}$ and $\mathrm{B}$ ). The result was a decrease in ejection fraction. In contrast those who underwent mitral valve replacement with chordal preservation had a significant decrease in left ventricular end diastolic and end systolic volumes. End systolic wall stress also fell significantly and the greater ejection fraction resulted in a smaller left ventricular size, reduced end systolic stress, and preservation of ejection performance despite closure of the low impedance left atrial ejection pathway.

Horstkotte and colleagues carried out a randomised trial in patients to compare valve replacement with total ablation of the subvalvar apparatus with replacement and posterior chordal preservation. ${ }^{25}$ Partial chordal preservation improved event free survival but the study did not answer the question whether preservation of both anterior and posterior chordae might achieve additional benefit (experimental studies had indicated that this might be so). This was addressed by Hennein et al who investigated patients with isolated mitral regurgitation after either conventional replacement with chordal transection, preservation of the posterior leaflet, or bi-leaflet preservation. ${ }^{20}$ For those in whom the chordae were excised, exercise capacity, left ventricular systolic dimensions, and cardiac index did not improve after valve replacement and left ventricular function deteriorated. Both the resting and exercise ejection fractions fell. In contrast exercise capacity improved after valve replacement in patients for whom the entire subvalvar apparatus was spared. Left ventricular systolic dimensions decreased, left ventricular function was maintained or improved, and resting ejection fraction was preserved. There was no significant difference between posterior chordal preservation alone and conservation of the entire subvalvar apparatus. This may be because the benefits of posterior chordal preservation alone leave little room for further improvement. Preservation of the anterior leaflet can also lead to left ventricular outflow obstruction. ${ }^{26}$

Okita and colleagues similarly compared preservation of both sets of chordae (during both mitral repair and replacement) with valve replacement and chordal transection. ${ }^{27}$ Again chordal preservation enhanced left ventricular function both immediately and late after surgery. Okita $e t$ $a l$ 's study also showed that chordal preservation, paradoxically, causes afterload to decrease rather than increase after mitral surgery. Chordal preservation appears to maintain ventricular shape and allows reduction in left ventricular volume. This reduces the radius term in the Laplace equation and consequently reduces wall stress. Decreased afterload reduces end systolic volume and in turn maintains ejection fraction at the preoperative level. The findings help to explain why mitral valve repair or replacement with preservation of the subvalvar apparatus leads to lower operative mortality and better long-term prognosis and quality of life than the conventional operation.

Analysis of data from the Veterans Administration Co-operative study on valvar heart disease shows that the preoperative predictors of normal ventricular ejection performance after mitral valve replacement are $(a)$ left ventricular ejection fraction $>0.50(b)$ left ventricular end systolic volume index $<50 \mathrm{ml} / \mathrm{m}^{2}$, and (c) absence of pulmonary hypertension. Most of our patients are in NYHA class III or IV and have deteriorated beyond these criteria. Most will not regain normal function status if replacement 
with chordal transection is performed. David's review of published reports on mitral valve surgery showed a collective hospital mortality of $0.9 \%$ for mitral repair compared with $7.3 \%$ for valve replacement. For the subgroup with chronic mitral regurgitation the corresponding figures were $3.4 \%$ for repair $v 10 \cdot 6 \%$ for replacement. ${ }^{16}$

\section{Effects of the annuloplasty ring}

Although mitral replacement with chordal preservation is equivalent to valve repair in terms of left ventricular function, deleterious effects are believed to occur because of fixation of the annulus with a rigid prosthesis. This may interfere with the distension and contraction of the basoconstrictor muscles which cause the normal annulus to contract during systole and dilate during diastole. The object of the annuloplasty ring is to correct annular dilatation in degenerative disease, increase coaption of the valve leaflets, and to prevent progressive annular dilatation. ${ }^{18}$ Two types of prosthetic valve ring are available. Carpentier's rigid prosthesis matches the systolic shape of the mitral annulus and creates a monocusp valve based on the anterior mitral leaflet. ${ }^{28}$ Flexible rings such as that of Duran or Messana Puig accommodate the normal changes in shape of the annulus during the cardiac cycle. ${ }^{29}$ These continuous changes in size and shape are accompanied by changes in the relative position of different parts of the annulus which are not in a single plane. Both rigid and flexible rings selectively reduce the posterior aspect of the annulus.

Experimental work has shown that the flexible ring acquires the systolic shape of the mitral valve during systole. ${ }^{29}$ During diastole it becomes circular and less stenotic. An elegant randomised study by David showed that fixation of the mitral annulus with a rigid ring impairs left ventricular function. ${ }^{30}$ Patients were randomised to either the Carpentier or the Duran annuloplasty ring. Those patients with the flexible Duran ring had improved preservation of left ventricular systolic function as assessed by pressure volume relation. Also the systolic anterior motion of the anterior leaflet (described repeatedly after insertion of a rigid ring) does not seem to occur with the flexible device. This potential cause of left ventricular outflow tract obstruction is probably the result of the rigid ring and base of the anterior leaflet being displaced towards the outflow tract during systole. In practice many valve repair procedures particularly for chordal rupture or ischaemic mitral regurgitation can be performed successfully without an annuloplasty ring and we prefer to avoid them.

\section{Conclusions}

Surgery for degenerative mitral regurgitation has become complex. Preservation of annulo-ventricular continuity through the chordae tendineae is an important determinant of operative survival, postoperative left ventricular function, long-term survival, and quality of life. Some cardiologists believe that NYHA I function is never achieved after conventional mitral replacement with chordal transection. Valve repair is the procedure of choice but when valve replacement is inevitable every effort should be made to preserve the posterior leaflet and its chordal attachments. Valve replacement with preservation of the subvalvar apparatus provides a functional outcome similar to that after valve repair but usually leads to life long anticoagulation.

Oxford Heart Centre,

fohn Radcliffe Hospital,

Headington,

Oxford OX3 9DU

1 Crawford MH, Souchek J, Oprian CA, Miller DC, Rahimtoola S, Giacomini JC, Sethi G, Hammermeister KE. Determinants of survival and left ventricular performance after mitral valve replacement. Circulation 1990;81: tricular p

2 Goldman ME, Mora F, Guarino T, Fuster V, Mindich BP. Mitral valvuloplasty is superior to valve replacement for preservation of left ventricular plasty is superior to valve replacement for preservation of left ventricular Am Coll Cardiol 1981:10:568-75.

3 Miki S, Kusuhara K, Ueda Y, Komeda M, Ohkita Y, Tahata T. Mitral valve Miki S, Kusuhara $\mathrm{K}$, Ueda $\mathrm{Y}$, Komeda $\mathrm{M}$, Ohkita $\mathrm{Y}$, Tahata $\mathrm{T}$. Mitral valve
replacement with preservation of chordae tendineae and papillary muscles. Ann Thorac Surg 1988;45:28-34.

4 Carabello BA. Preservation of left ventricular function in patients with mitral regurgitation: A realistic goal for the nineties $\mathcal{F}$ Am Coll Cardiol 1990;15: $564-5$

5 Bonchek LI, Olinger GN, Siegel R, Tresch DD, Keelan MH. Left ventricular performance after mitral reconstruction for mitral regurgitation. $\mathcal{f}$ Thorac Cardiovasc Surg 1984;88:122-7.

6 Cohn LH, Kowalker W, Bhatia S, et al. Comparative morbidity of mitral valve repair versus replacement for mitral regurgitation with and without coronary artery disease. Ann Thorac Surg 1988;45:284-90.

7 Figueras MG, Lugne I, Montero A, et al. A comparison of replacement and reconstruction in patients with mitral regurgitation. F Thorac Cardiovasc Surg 1987;93:665-74.

8 Sand ME, Naftel DC, Blackstone EH, et al. A comparison of repair and replacement for mitral insufficiency. F Thorac Cardiovasc Surg 1987;94: 208-19.

9 Orniston JA, Shah PM, Tei C, et al. Size and motion of the mitral valve in man. I. A two-dimensional echocardiographic method and findings in normal subjects. Circulation 1981;64:113-20.

10 Gams E, Heimisch W, Hagl S, et al. Significance of the subvalvular apparatus following mitral valve replacement [abstr]. Circulation 1987;76(suppl following

11 Hansen DE, Cahill PH, Derby GC, et al. Relative contributions of the anterior and posterior mitral chordae tendineae to canine global left ventricular sysand posterior mitral chordae tendineae to canine global
tolic function. $\mathcal{F}$ Thorac Cardiovasc Surg 1987;93:45-55.

12 Krayenbuehl HP. Surgery for mitral regurgitation. Repair versus valve replacement. Eur Heart $\mathcal{f} 1986 ; 7: 638-43$.

13 Schuler G, Peterson KL, Johnson A, et al. Temporal response of left ventricular performance of mitral valve surgery. Circulation 1979;59:1218-30.

14 Hetzer R, Bougioukas G, Franz M, Borst HG. Mitral valve replacement with preservation of papillary muscles and chordae tendineae-revival of a seemingly forgotten concept: I Preliminary clinical report. Thorac Cardiovas Surg 1983;31:291-6.

15 Miller DW, Johnson DD, Ivey TD. Does preservation of the posterior chordae tendineae enhance survival during mitral valve replacement? Ann Thorac Surg 1979;28:22-7.

16 David TE. A rational approach to the surgical treatment of mitral valve disease. In: Karp RD, ed. Advances in cardiac surgery, vol 2. Chicago: Mosby Year Book 1990;63-84.

17 Cooley DA, Ingram MT. Intravalvular implantation of mitral valve prostheses. Tex Heart Inst $\mathcal{f} 1987 ; 14: 188-93$.

18 Asano K, Furuse A. Techniques of modified mitral valve replacement with preservation of the posterior leaflet and chordae tendineae. Thorac Cardiovasc Surg 1987;35:206-8.

19 Miki S, Kusuhara K, Ueda Y, Komeda M, Ohkita Y, Tahata T. Mitral valve replacement with preservation of chordae tendineae and papillary muscles.

20 Hennein HA, Sawain JA, McIntosh CL, Brown RO, Stone CD, Clark RE. Comparative assessment of chordal preservation versus chordal transection during mitral valve replacement. $¥$ Thorac Cardiovasc Surg 1990;99:228-37.

21 Lillehei CW, Levy MJ, Bonnabeau RC. Mitral valve replacement with preservation of papillary muscles and chordae tendineae. $\mathcal{f}$ Thorac preservation of papillary musces
Cardiovasc Surg 1981;24:236-9.

22 David TE, Straus HD, Mesher E, Anderson MJ, MacDonald IL, Buda AJ. Is it important to preserve the chordae tendineae and papillary muscles during mitral valve replacement. Can $\mathcal{F}$ Surg 1981;24:236-9.

23 David TE. Mitral valve replacement with preservation of chordae tendineae. Rationale and technical consideration. Ann Thorac Surg 1986;41:680-2.

24 Rozich TD, Carabello BA, Usher BW, et al. Mitral valve replacement with and without chordal preservation in patients with chronic mitral regurgitation. Circulation 1992;86:1718-26.

25 Horstkotte D, Hager D, Schulte WB, Strauer BE. The effect of chorda preservation on late outcome after mitral valve replacement. A randomised study. I Heart Valve Dis 1993;2:150-8.

26 Mihaileanu S, Marino JP, Chauvaud S, et al. Left ventricular outflow obstruction after mitral valve repair (Carpentier's technique). Proposed mechanisms of disease. Circulation 1988;3:78-84.

27 Okita Y, Miki S, Veda Y, et al. Comparative evaluation of left ventricular performance after mitral valve repair or valve replacement with or without chordal preservation. $\mathcal{F}$ Heart Valve Dis 1993;2:159-66.

28 Carpentier A, Deloche A, Dauptain J, et al. A new reconstructive operation for correction of mitral and tricuspid insufficiency. F Thorac Cardiovasc Surg 1971;61:1-13.

29 van Rijk-Swikker GL, Mast F, Schipperheyn JJ, et al. Comparison of rigid and flexible rings for annuloplasty of the porcine mitral valve. Circulation $1990: 82.58-64$

30 David TE, Komeda M, Pollick C, et al. Mitral valve annuloplasty. The effect of the type on left ventricular function. Ann Thorac Surg 1989;47:524-7. 